\title{
Japan and Its Rapid Ageing Society: Does e-health Technology Provide a Solution?
}

Susanne Brucksch*

German Institute for Japanese Studies Tokyo (DIJ), Jochi Kioizaka Bldg. 2F, 7-1 Kioicho, Chiyoda-ku, Tokyo 102-0094, Japan

*Corresponding author: Susanne Brucksch, German Institute for Japanese Studies Tokyo (DIJ), Jochi Kioizaka Bldg. 2F, 7-1 Kioicho, Chiyoda-ku, Tokyo 102-0094, Japan, E-mail: brucksch@dijtokyo.org

Received date: August 08, 2018; Accepted date: August 29, 2018; Published date: August 31, 2018

Copyright: $\odot 2018$ Brucksch S. This is an open-access article distributed under the terms of the Creative Commons Attribution License, which permits unrestricted use, distribution, and reproduction in any medium, provided the original author and source are credited.

\begin{abstract}
Japan can be called a rapidly ageing society. In 2016, one-quarter of the Japanese society was aged 65 and over. Against this backdrop, in this article I argue that a rapidly ageing society like Japan will rely heavily on technological solutions to ensure adequate care and a decent standard of living for elderly people. Thus, the country is predestined as a case study considering the relationship between ageing, professional care, e-health technology and other assistive devices. I use examples from Telehealth networks and monitoring sensor systems to shed some light on these recent and growing developments in the field of e-health technologies.
\end{abstract}

Keywords: Demographic shift; Homecare; Elderly care; Monitoring sensor systems; Telehealth networks

\section{Introduction}

Japan can be called a rapidly ageing society. In 2016, one-quarter of the Japanese society was aged 65 and over. The $\mathrm{CaO}$ estimates that one-third of the population will be 65 or older by 2025 [1]. Although the country has a shrinking population, its life expectancy is the highest among all the OECD countries (J: 83.9 years at birth and OECD average: 80.6 years in 2015) while its fertility rate remains far below replacement level (J: 1.5 and OECD average: 1.7 children per woman in 2015). Nevertheless, the demographic shift is not an isolated Japanese phenomenon. One can say that the country is at the forefront of this trend among the OECD member states [2,3]. Against this background, I argue that a rapidly ageing society like Japan will rely on technological solutions to ensure adequate care and a decent standard of living for elderly people. Thus, the country is predestined as a case to study the relationship between ageing, professional care, e-health technology and other assistive devices. In the following paragraphs, I will explain in greater detail this argument.

\section{Rapid ageing and its ramifications in Japan}

In an ageing population, healthcare costs will increasingly become a decisive factor in sustaining an adequate level of care for the elderly. Statistically, 5.8 people (aged between 20 and 64) supported on average one elderly person (aged 65 and over) in 1990, and 2.2 people in 2016, barely 1.9 people will carry the financial burden for one elderly person in 2025 [1]. In Japan, healthcare expenditures are covered by both the citizens' health insurance (kokumin kenkō hoken) and additional health insurances, municipalities and prefectures, a larger share of which comes from tax revenues and cross-subsidization [4]. In addition, the long-term care insurance (LTCI) was introduced in 2000, which has a focus on covering the costs of elderly care, which used to be reimbursed mainly by the citizens' health insurance. Furthermore, overall healthcare expenditures have soared from 3.6 million yen in 2000 to 10.4 trillion yen in 2016 and are expected to reach 21.0 trillion yen by 2025, according to recent calculations [5]. Specifically, the cost for medical and elderly care is expected to rise along with the rising number of elderly people in more frail conditions (age group of 75+) and the need for professional caregivers. Here, we can see one of the main reasons for the national government and local communities to focus increasingly on cost reduction. Accordingly, the MHLW and government have begun to favour health preservation, active lifestyle for elderly people and homecare without neglecting investment in required institutionalized care for people in more frail conditions [6-10]. These measures are included with the promotion of technological appliances and e-health technologies.

A further factor supporting this drive to technological solutions is the growing gap of professional care workers in Japan. As demonstrated in Figure 1, the number of long-term-care professionals increased from 550,000 in 2000 to 1.5 million in 2012 [11]. Additionally, the number of care personnel will rise further to about 2.3 million in 2025 while the need for long-term-care professionals will become 2.5 million that very same year. This implies a shortage of 160,000 care workers. However, the latest follow-up investigation in 2015 revealed that the need for professional care staff had increased even further to an estimated demand for care staff of around 2.53 million by 2025 yet the available number of care workers will only be 2.15 million. That is a gap of 380,000 care workers for that year [12]. Therefore, Japan is very likely to struggle in the near future with a tremendous labour shortage in long-term care but also medical care. What is more, the additional training and recruitment of a higher number of professional care staff will allegedly not suffice to close the gap between necessary and available workforce in medical and elderly care over next few years. This gap may not close even if Japan succeeds in attracting a higher share of healthcare staff from foreign countries such as the Philippines due to the government caps on the number of foreign works allowed to migrant into Japan [13]. Hence, there is an urgent need for solutions, with respect to care professionals and/or assistive technologies. The picture becomes even direr if we consider the peripheral regions of Japan. 


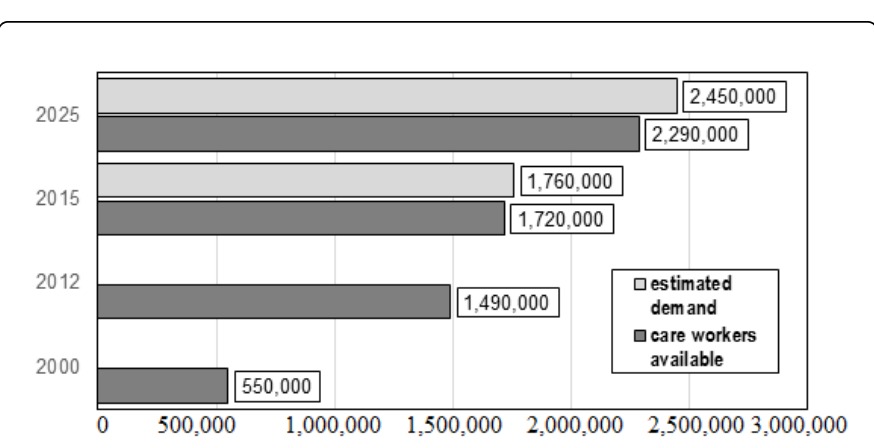

Figure 1: The number of professional long-term-care workers in Japan

Another reason is that rapidly ageing, population decline and labour shortages in healthcare are most evident in peripheral regions [14]. In particular, local communities in rural areas struggle with problems such as shortages of physicians and specialists in obstetrics and pediatrics, emergency room availability, closure of hospitals and shortages of nursing staff $[4,15]$. Due to this background, the introduction of healthcare programs employing information and communication technology (ICT) are currently in consideration as one solution to create and sustain an adequate health and long-term care infrastructure in peripheral regions. For instance, there are some good examples, such as an ICT-based health maintenance program in Mitsuke City, Niigata Prefecture [16]. Specifically, the average physical strength of participants aged 65 and over was evaluated at the beginning of the program as that of someone aged 65.4 years, according to medical stress tests and physical activities. After three months of participation in ICT-induced activities for health maintenance, the average physical strength had improved to that of someone 60.9-years-old. Moreover, the health expenditures of participants in the four years during and after the program were 104,234 yen lower than for non-participants, according to a study by the Ministry of Internal Affairs and Communications.

However, the diffusion of ICT for healthcare is not yet very widespread. According to the Ministry of Land, Infrastructure, Transport and Tourism, there were still a small number of clinics and hospitals in peripheral areas equipped with ICT networks and most of these used rather simple applications at that time [17]. The issue of sustaining an adequate healthcare infrastructure and network of professional services are the most critical at the local level.

Japan's government, as well as local communities have taken several measures to tackle the issues of rapid ageing, population decline, growing healthcare spending and financial constraints. One objective is to expand the proportion of aged people that continue to live at home into higher ages. Interestingly, a survey conducted in Japan among people over 55-years-old shows that the majority (54.6\%) prefers to stay at home until the end of their lives [6]. In comparison, a quarter (27.7\%) said they wished to stay in a hospital (including hospices), and just under one-tenth of respondents $(8.6 \%)$ preferred care facilities. In other words, there is a wide preference for homecare solutions and autonomy into higher ages. Due to the low number of children per family in Japan, family or spousal care is not an option in many cases. To prevent or delay their dependence on long-term care as long as possible, the Ministry of Health, Labor, and Welfare of Japan (MHLW) supports domestic healthcare through the promotion of technical advancements in terms of R\&D and purchase via the LTCI.

It is also important to note that female caregivers shoulder most of the care work in Japan and that lower back pain is one of the most frequent occupational diseases in long-term care [15]. For instance, $42.4 \%$ of male and $56.6 \%$ of female outpatients reported lower back damage. Specifically, lower back damage is one of the most prevalent diseases among women, and is far less frequent among men in Japan, according to the "Basic survey of Japanese people's lifestyles" (Kokumin seikatsu kiso chōsa) published by the MHLW [18]. Accordingly, these numbers underline the necessity of some kind assistive technology in elderly care such as transfer aids to prevent an additional burden on caring staff when moving bedridden or frail people to keep them mobile or to avoid bedsores. During an interview study, however, we received the feedback that transfer is a difficult task and yet lifts are not common in most care facilities and private houses in Japan, since they frequently need too much space. Instead, care giving relatives and nursing staff have to lift disabled people themselves, which leads to back damage and psychological stress [19]. In other words, with the rapid growing share of aged people 75 years and older, the necessity for assistive technologies will rise fast as care giving relatives and professional care workers need assistance in providing support to patients in frail or bedridden conditions. These assistive technologies encompass smart robotic devices and e-health technologies, which increasingly utilize GPS or monitoring function to prevent falls of aged people living at home and to trigger remote alert to care personnel (see below for some more details).

\section{E-health in Japan}

The rapidly ageing population issue has tremendous ramifications, ones that lead to strong incentives to innovate healthcare technology for aged people. In the field of e-health technology, two recent developments in Japan are worth mentioning: monitoring sensor systems (mimamori sensā) and ICT-based Telehealth networks being implemented in various locations throughout Japan. Carretero lists smart homes, generic ICT products, as well as services and applications with assistive technology and others to create a way to support an "independent living of aged people" [20]. Accordingly, monitoring sensor systems in smart houses and ICT-based Telehealth networks can be included in the field of e-technology goals for aged people.

Monitoring sensor systems attract attention because they are expected to integrate the following characteristics: monitoring multiple rooms at the same time (e.g. bathrooms), allowing for help not to be solely dependent on the care-receivers voluntary actions, sending an alarm to caregivers if their care-receiver falls, sending information to caregivers after changes in health of the care-receiver is detected, or even having the potential of monitoring people with dementia (AIST n.y.). A number of Japanese manufacturers have made efforts to come up with sensor technologies and smart housing solutions specifically directed to healthy, elderly residents. For instance, Sekisui House is one of these manufacturers, and also one of Japan's largest house building companies. They have recently developed a Smart Healthcare House with a monitoring sensor system, an electronic and computercontrolled integrated systems within the home, which includes a monitoring sensor network that enables daily health checks and supports the autonomous living of aged people [21,22]. Putting it differently, smart house solutions have recently entered the domain of homecare technology for people in higher age's ranges. 
Page 3 of 4

The introduction of ICT-based Telehealth systems is another measure implemented by local and prefectural governments, which is also a political goal supported by the MHLW. Cowan and TurnerSmith have identified Telehealth systems as relevant in the context of "reversal and amelioration of the declining capacities of older people" [23]. In our interview study, we examined two cases of Telehealth networks in peripheral regions in Japan, one in Fukui Prefecture at the regional level and one of Iida City and the neighbouring Shimoina District at the local level in Nagano Prefecture. Both regions are affected by particularly rapid ageing, declining population and shortages of financial resources and professional healthcare. In order to address rising healthcare spending and shortages of healthcare professionals, they introduced Telehealth networks to reduce the number of hospitals and to maintain an adequate healthcare infrastructure despite geographical distances between urban centers and remote places particularly in mountainous and rural regions [19]. Both networks link institutions in the domain of medical care, including hospitals, clinics, rehabilitation centers and aftercare at home as well as the domain of elderly care and homecare, including care managers, care organizations, day care centers with care-receivers and their families through information and communication technology (ICT).

Although there are e-health technologies and smart devices that still face various obstacles such as user reluctance or are not being ready yet for implementation, we must bear in mind that manufacturers are in the midst of a search for suitable technologies that have many steps to follow until a feasible prototype and applicable product will be marketready. For instance, the developers of the monitoring sensor system we examined reported that users were reluctant to use a patch-type sensor attached to the skin; instead they preferred a contactless system that had the same level of accuracy and reliability in detecting bioinformation. The developing team also faced regulatory restrictions under the Medical Practitioners' Act of Japan as recommendations cannot be too specific, since they must not substitute a diagnosis by a medical professional [19]. Consequently, developers face high security expectations from their caregiving and care-receiving users and strict standards to receive approval for their products from authoritative bodies if they choose to enter the domain of healthcare.

We found similar concerns about implementing ICT-based Telehealth systems voiced by medical professionals and care workers, who were concerned about workload, ease of use and system literacy. However, none of the reported concerns seemed severe enough to prevent the establishment of these systems in the long run. In other words, practicality appears to be a contributing factor, while the complexity of the e-health system is the inhibiting factor for a successful implementation. However, this can be improved by appropriate training among healthcare professionals in proper protocols and procedures. Besides, e-health and other data processing technology raise concerns regarding privacy and data security. In both Telehealth networks examined, data security was managed through limited access by strict user registration, patients' or care-receiver's prior permission to enter their records along with different levels of access based on professional status and regulatory requirements. Although there was hardly any hesitation reported by patients and care-receivers, concerns about data security and privacy have been taken into account, as patients' permission needs to be granted to and can be withdrawn from each healthcare institution having access to their health records [19].

\section{Conclusion}

So, if we elaborate on e-health technologies and any other technical solution we might jump to the conclusion questioning whether such technologies are useful. In the beginning, however, I have argued that a rapidly ageing society like Japan will rely heavily on technological solutions to ensure adequate care and a decent standard of living for elderly people. Although the demand for fully-trained professional care staff, drawn both from domestic and foreign sources, is likely to increase considerably, it is unlikely there will exist enough elderly care workers in the future to satisfy that demand. Due to this and other reasons mentioned above, e-health technologies and assistive devices will be needed for Japan's rapid ageing society. For this reason, I conclude there is a demand and, therefore, sufficient incentives for manufacturers to invest in R\&D activities for feasible devices and ehealth appliances. In other words, these appliances will contribute to the autonomy of aged people who continue living at home while Telehealth networks in peripheral regions will function to sustain an adequate healthcare infrastructure in peripheral regions that are affected by the effects of demographic ageing and labour shortages in health and elderly care.

\section{Acknowledgement}

This article refers to data that are the outcome of a joint research project between the Leiden Asia Centre and the German Institute for Japanese Studies (DIJ). The project was initiated and financially supported by Leiden Asia Centre under their research scheme "Aging Japan: Leading the way into the future". The project on "Ageing in Japan: Domestic Healthcare Technologies in Japan" was conceptualized and conducted at the German Institute for Japanese Studies (DIJ) partially under its research scheme of "The Future of Local Communities in Japan-Risks and Opportunities in the Face of Multiple Challenges".

\section{References}

1. Cabinet Office $(\mathrm{CaO})$ (2017) Heisei 29-nenban kōrei shakai hakusho. Dai-isshō Kōrei-ka no jōkyō. [White Paper on the Aging Society 2017: The Aging Situation]. Government of Japan.

2. Organisation For Economic Co-Operation And Development (OECD) (2017) Health at a Glance 2017: OECD Indicators: How Does Japan Compare?.

3. Organisation For Economic Co-Operation And Development (OECD) (2018) Data: Elderly Population.

4. Campbell JC, Ikegami N, Tsugawa Y (2014) The political-historical context of Japanese healthcare. In: N. Ikegami (ed.): Universal Health Coverage for Inclusive and Sustainable Development: Lessons from Japan. World Bank Group, Washington, USA, pp.15-26.

5. Ministry of Health, Labor and Welfare (MHLW) (2015c) Kōteki kaigo hoken seido no genjō to kongo no yakuwari [Present Situation and Future Role of the Public Care Insurance System]. Government of Japan.

6. Cabinet Office (CaO) (2012) Kōreika no kenkō ni kansuru ishiki-chōsa [Results of the Survey about Health Consciousness among Elderly People]. Government of Japan.

7. Cabinet Office $(\mathrm{CaO})$ (2014) Kōreika no jōkyō [Aging Situation]. Government of Japan.

8. Cabinet Office $(\mathrm{CaO})$ (2016) Chi'iki no keizai 2016-Jinkō genshō mondai no kokufuku [Regional Economy 2016. For Overcoming the Challenges from Population Decline]. Government of Japan.

9. Ministry of Health, Labor and Welfare MHLW (MHLW) (2015b) Vital Statistics: E-Stat. Government of Japan. 
Citation: Brucksch S (2018) Japan and Its Rapid Ageing Society: Does e-health Technology Provide a Solution?. J Aging Sci 6: 192. doi: $10.4172 / 2329-8847.1000192$

Page 4 of 4

10. Ministry of Finance (MOF, Japan) (2016) Nihon no zaisei-kankei shiryō [Sources about Japanese Financial Affairs]. Government of Japan.

11. Ministry of Health, Labor and Welfare (MHLW) (2014) Kaigo jinzai no kakuho ni tsuite. Dai ikkai fukushi jinzai kakuho taisaku kenshō-kai (H26.6.4), shiryō 2 [Securing Human Resources in Long-term Care. First Meeting about Measures to Secure Human Resources in the Field of Welfare]. Government of Japan.

12. Ministry of Health, Labor and Welfare MHLW (Ministry of Health, Labor and Welfare) (2015a) 2015-nen ni muketa kaigo jinzai ni kakaru jukyū suikei (kakutei-chi) [Demand and Supply of Care Personnel until 2025 (Definitive Value)]. Government of Japan.

13. Vogt G (2017) In: Vogt G (ed.) Population Aging and International Health-Caregiver Migration to Japan. Springer International Publishing, Zurich, Switzerland.

14. National Association of Trade Promotion for Small and Medium Enterprises (2014). White Paper on Small and Medium Enterprises in Japan. Fight Song for Micro Businesses.

15. Campbell J C (2014) Japan's Long-Term Care Insurance Program as a Model for Middle-Income Nations. In: N. Ikegami (ed.) Universal Health Coverage for Inclusive and Sustainable Development: Lessons from Japan.World Bank Group, Washington, USA pp.57-67.

16. Ministry of Internal Affairs and Communications (MIC) (2013) Heisei 25-nendo-ban Jōhō tsūshin Hakusho [White Paper on Information and Communication 2013]. Government of Japan.

17. Ministry of Land, Infrastructure, Transport and Tourism (MLIT) (2015) White Paper on Land, Infrastructure, Transport and Tourism in Japan Government of Japan.
18. Ministry of Health, Labor and Welfare (MHLW) (2017) Heisei-28-nen. Kokumin seikatsu kiso chōsa [Basic survey of Japanese people’s lifestyles 2016]. Government of Japan.

19. Brucksch S, Schulz F (2018) In: Brucksch S, Schulz F (eds.) Ageing in Japan. Domestic Healthcare Technologies. A Qualitative Interview Study on Care Robots, Monitoring Sensor Systems, and ICT-based Telehealth Systems. Leiden Asia Centre (LAC) and German Institute for Japanese Studies Tokyo (DIJ) Tokyo, Japan.

20. Carretero S (2015). Technology-enabled Services for Older People Living at Home Independently: Lessons for Public Long-term Care Authorities in the EU Member States. In: JRC Scientific and Technical Reports Series. Institute for Prospective Technological Studies, European Commission, Joint Research Centre, Seville, Spain.

21. Sekisui House (2017) Katsudō 1: "Netto. Zero. Enerugī. Hausu" o kakudai [Expanding on "Houses with Internet and Zero Energy (Consumption)].

22. Nikkei (2015) Kuchō mo kenkō mo kanri. Sekisui ga jisedai sumāto hausu [Sekisui (Introduces) Next Generation Smart House (that) Monitors Health and Climate].

23. Cowan D, Turner-Smith A (1999) The Role of Assistive Technology in Alternative Models of Care for Older People. In: Suderland (ed.) With Respect to Old Age. Long-term Care-Rights and Responsibilities. HMSO, London, UK, pp. 325-346.
This article was originally published in a special issue, entitled: "Aging Challenges in a Digital World", Edited by Dr. Fereshteh Barei, Paris Dauphine University, Paris, France. 